\title{
Extensification de la conduite des prairies et entretien de l'environnement dans le parc naturel regional de Brenne par des élevages bovins charolais allaitants
}

\author{
R. Le Dorze ${ }^{\mathrm{a}}$, J.L. Roy ${ }^{\mathrm{a}}$, M. Frain ${ }^{\mathrm{b}}$, F.X. de Montard ${ }^{\mathrm{c}}$ \\ a Chambre d'agriculture de l'Indre, 36300 Le Blanc ; ${ }^{b}$ ADER Auvergne, 5, rue Montrognon, \\ 63100 Clermont-Ferrand ; "Inra agronomie, av. du Brézet, 63039 Clermont-Ferrand, \\ France
}

La Brenne est une petite région naturelle du nord-ouest du Massif Central, parsemée de prairies et d'étangs avec quelques bois ; les sols, formés sur grès, sont peu profonds à dominante sableuse. L'élevage charolais est l'activité agricole dominante mais se renouvelle difficilement car les terres sont recherchées pour constituer chasses, étangs et plantations médiocres de pin sylvestre. Les éleveurs ne peuvent agrandir à la fois terres et troupeaux et les nouveaux propriétaires préfèrent laisser en friche. Outre le maintien d'un paysage ouvert et varié, l'élevage bovin est un facteur favorable pour l'alimentation du gibier et la fécondité des étangs. L'extensification permettrait d'entretenir l'espace au moindre coût. Une expérimentation d'extensification à 0,58 UGB $\cdot h^{-1}$ dans trois unités de 18 ha a montré qu'en trois ans le pâturage permettait de réduire la progression des ronciers à $6,4 \%$ de la surface pâturable contre $11,8 \%$ observés dans les friches clôturées et totalement inexploitées. Le broyage des ronciers en quatrième année, puis un peu plus tard, le dépôt de bottes de foin au milieu de leurs repousses a permis de ramener le bilan des pertes de surface à 5,4 \% en cinq ans contre $19,1 \%$ dans les friches. Les performances des veaux ont été identiques à celles des veaux des fermes de référence, mais la marge brute a diminué de $1500 \mathrm{~F} \cdot \mathrm{ha}^{-1}$ en raison du chargement plus faible, ce qui pose la question de la rémunćration de cet entretien.

\section{Compétition racinaire et utilisation de la réserve en eau dans une association de noisetier et de dactyle}

\author{
F.X. de Montard
}

Inra agronomie, 234, av. du Brézet, 63039 Clermont-Ferrand, France

La compétition racinaire et l'utilisation de la ressource en eau ont été étudiées dans une association noisetier-dactyle par comparaison avec un dactyle sans arbres et une plantation de noisetiers entièrement desherbée depuis trois ans. La croissance cumulée du tronc et le volume de la couronne sont plus de deux fois supérieurs pour les arbres desherbés, mieux alimentés en eau $(p<0,01)$. La production de dactyle n'est affectée par la présence des arbres qu'en période de stress hydrique et dans un rayon d'un mètre autour du tronc, soit $-30 \%$ et $-24 \%$ en troisième et quatrième année $(p<0,05)$. Le dactyle sans arbres est d'abord moins productif $(-25 \%, p<0,05)$ que le dactyle abrité mais la tendance s'inverse au cours du quatrième été lorsque la transmission de la lumière est réduite de $30 \%$. Les croissances racinaires observées derrière une vitre verticale placée à $90 \mathrm{~cm}$ du tronc, ne sont pas différentes pour le noisetier désherbé et le noisetier enherbé, ce dernier déplaçant néanmoins son activité racinaire de $20 \mathrm{~cm}$ en profondeur. Le bilan des consommations d'eau est peu différent entre l'arbre en sol nu et l'association arbre+herbe, ce qui indique une sévère compétition pour la ressource hydrique dans l'association. Les potenticls hydriques montrent une amélioration de l'état hydrique pour l'arbre et une dégradation pour l'herbe à mesure que les racines de l'arbre s'installent en profondeur empêchant la descente des nouvelles racines de l'herbe tant que le profil n'est pas réhumecté. 